\title{
An Improved Algorithm for 3D NoC Floorplanning Based on Particle Swarm Optimization of Nesting Simulated Annealing
}

\author{
Song Guozhi ${ }^{1}$, Zhang Dakun ${ }^{1, *}$, Huang $\mathrm{Cui}^{1}$ and Wang Lianlian ${ }^{2}$ \\ ${ }^{1}$ School of Computer Science and Software Engineering, Tianjin Polytechnic University, Tianjin300387, China; \\ ${ }^{2}$ Network Center, TianJin Agricultural University, Tianjin 300384, China
}

\begin{abstract}
In this paper, an improved floorplanning algorithm, named the floorplanning algorithm based on particle swarm optimization algorithm nesting simulated annealing to optimize the floorplans (PSO-SA-NoC), has been proposed with simulations conducted to verify this algorithm. The simulation results are compared with the original Simulated Annealing-NoC. The results show that the CPU's process time of the PSO-SA-NoC algorithm decreased by $35.39 \%$. The packet transmission latency reduces $4.05 \%$ in the average case and $83.3 \%$ in the best case respectively. The throughput improves $1.72 \%$ in the average case and $10.57 \%$ in the best case respectively.
\end{abstract}

Keywords: 3D NoC, floorplanning algorithm, heterogeneous floorplans, PSO, SA.

\section{INTRODUCTION}

Three-dimensional network on chip (3D NoC) has become one of the latest hot interdisciplinary research topics involving both integrated circuit (IC) and computer science. It was developed on the basis of integrated circuit (IC), system on chip (SoC) and on-chip network (2D NoC). The main problem solved is on-chip communication bottleneck caused by high density integration. Nowadays, because of the shorter global interconnections, higher performance, lower interconnect losses, higher packing density, smaller size and many other advantages, 3D NoC has drawn more and more attention from both industry and academia.

A floorplan of an IC is a schematic representation of tentative placement of major functional IP blocks in Electronic Design Automation (EDA). Floorplans can be categorized into two groups, the sliceable floorplans [1-3] and nonsliceable floorplans [4-7]. For examples, a team from Tsinghua University led by She-Qin Dong mainly focused on the buffer insertion algorithm for interconnect centric floorplanning [8] and 3-D floorplan representation based on the methodologies of Corner Block List (CBL) [9]. Their contribution also included a divide-and-conquer 2.5-D floorplanning algorithm [10]. Liang-Li He et al. in [11] conducted a thorough study of virtual layout and proposed a FOB based man-machine interactive loading layout method. Another team led by Young, Evangeline F.Y. from Hong Kong university of science and technology delved into the problem of bus-driven floorplanning [12] and 3-D floorplanning using labeled tree and dual sequences [13]. Jingjing in [14] proposed a space feature optimization based mapping layout algorithm and they make a great contribution on energy consumption optimization of three dimensional networks-on-chip. Kakoee Mohammad Reza and Angiolin Federico et al. in [15] proposed a floorplan-aware toolchain for $\mathrm{NoC}$ design and synthesis integrated with a graphical front-end. They showed that not only a great amount of time and effort can be saved thanks to the easy-to-use proposed environment, but also that the quality of the final netlist can be improved due to the optimizations unlocked by the earlystage interaction among the designer and the proposed toolchain. They presented a floorplan-aware toolchain for NoC design and synthesis integrated with a graphical front-end, The resulting design methodology is highly automated yet entails rich interaction with the user, spanning across traffic flow specification, topology synthesis and physical floorplanning, with back-annotation capabilities and opportunities for incremental design. de Paulo, V and Ababei, C worked on homogeneous networks over heterogeneous floorplans [16]. They proposed a design methodology consisting of floorplanning and router assignment in a specifically designed tool that integrates a cycle accurate NoC simulator. It is implemented and then used to investigate the new architecture and show that experimental results are application specific with potential significant performance improvements for some testcases.

In 1983, Kirkpatrick proposed modern Simulated Annealing (SA) algorithm, and used the algorithm to solve Large-scale combinatorial optimization problem successfully [17]. Yurong Feng in Kunming University applied SA to web process and current control in zinc, which achieved good results from the test in saving electricity costs of zinc plant [18]. This benefited from the efficiency and superiority of SA algorithm. Lin Wang in Inner Mongolia University of Technology applied SA to optimize the design of T-shaped micro-reactor [19]. SA has also been applied to assessment of dynamic positioning capability by Zhengfeng Liu in China Ship Scientific Research Center [20]. In 1995, Dr. James Kennedy and Russell Eberhart proposed Particle Swarm Optimization (PSO) algorithm [21, 22], and then 
PSO is widely used in various fields of social life and scientific research. Guangqing Bao in Lanzhou University of Technology applied PSO algorithm in wind power system [23]. In addition, PSO has also been applied to the optimize scheduling of the reservoir [24] and vehicle routing problem [25]. Li Jiang in Anhui University applied PSO and SA to study of BP network learning method [26]. In this paper, we first apply the classical PSO together with SA to 3D NoC floorplanning algorithm and open a new field of PSO and SA application.

The rest of the paper is organized as follows. In Section 2, we specify the design and realization of PSO-SA-NoC algorithm. In Section 3, we conduct simulation experiments using VNOC3 Simulator to test the degree of improvement of the proposed algorithm on CPU processing time, average flit latency and network throughput. Finally, Section 4 is the conclusion of this paper and the future work we can do.

\section{FLOORPLANNING ALGORITHM BASED ON PARTICLE SWARM OPTIMIZATION ALGORITHM NESTING SIMULATED ANNEALING ALGORITHM TO OPTIMIZE THE FLOORPLANS}

\subsection{PSO-SA-NoC Algorithm Design}

In this paper, an improved floorplanning algorithm named the floorplanning algorithm based on particle swarm optimization algorithm nesting simulated annealing to optimize the floorplans (PSO-SA-NoC) has been proposed. The algorithm is an improvement of the original floorplanning algorithm based on Simulated Annealing (SA) algorithm to optimize the floorplans, using both the parallel computing features of PSO and the global optimization features of SA to mainly optimize average flit latency and network throughput.

The cost function of PSO-SA-NoC algorithm used is shown in Formula (1),

Costfunction $=\alpha \cdot$ Area $+(1-\alpha)$ WireLength

In (1), Costfunction represents the cost of completing the layout needs. Area represents the layout area used by IP cores layout using. Length represents length of the connection of generating layout uses. $\alpha$ is a parameter specified by the user which used to balance the area of tiles and the length of layout uses and valued from 0 to 1 . In our algorithm, the value of $\alpha$ is 0.25 . The update formulae of velocity and position in our algorithm are shown as Formula (2) and Formula (3),

$$
\begin{aligned}
& v_{i+1}=\omega v_{i}+c_{1} \cdot d_{1}\left(\text { pre }_{-} \cos t_{i}-x_{i}\right)+c_{2} \cdot d_{2}\left(\text { best }_{i}-x_{i}\right) \\
& x_{i+1}=x_{i}+v_{i}
\end{aligned}
$$

In Formula (2), inertia weight $\omega$ uses variable weight and calculated formula is shown as Formula (4). The initial value of $\omega_{\min }$ and $\omega_{\max }$ are 0.5 and 3 . The parameter count represents the current number of iterations and $N$ represents the maximum number of iterations. Learning factor $c_{1}$ and $c_{2}$ use synchronous time-varying way and the calculated formula is shown as Formula (5). The initial value of $c_{\min }$ and $c_{\max }$ are 0.25 and 3 . The parameter count represents the current num- ber of iterations and $N$ represents the maximum number of iterations. Random parameters $d_{1}$ and $d_{2}$ are numbers that program randomly generated from 0 to 1 . The parameter pre_cost $t_{i}$ represents the best position of the particle itself and best $_{i}$ represents the optimum position of particle swarm, wherein the velocity is in the range $[3,3]$. If the value exceeds the determined range, then revise it to equal to the maximum. The position sequence of particles satisfies with binary code. It also needs to be revised if out of the determined range. If the location information obtained is less than 0.5 , then record it as 0 , else record it as 1 .

$$
\begin{aligned}
& \omega=\omega_{\max }-\frac{\left(\omega_{\max }-\omega_{\min }\right) * \text { count }}{N} \\
& c_{1}=c_{2}=c_{\max }-\frac{\left(c_{\max }-c_{\min }\right) * \text { count }}{N}
\end{aligned}
$$

The simulated annealing parameters used in this algorithm include annealing probability $p$ and the calculated formula is shown as Formula (6), in which pre $i]$ represents the fitness value of each particle and $T$ represents the initial temperature.

$$
p=\exp \left(\frac{\operatorname{pre}[i]-p r e \_\cos t}{T}\right) \quad i \in[0, \operatorname{sh} u]
$$

The given initial value of final temperature term temp is 0.1 . The annealing formula is shown as Formula (7), in which $r_{-} t$ represents temperature control parameter, $\lambda$ is specified by users and the default value is 1.3 , and $s v$ represents the length of number of particles which have calculate fitness value.

$$
T=r_{-} t^{*} T, \quad r_{-} t=\exp \left(\frac{\lambda * T}{s v}\right)
$$

\subsection{PSO-SA-NoC Algorithm Realization}

In this part, we show the realization of PSO-SA-NoC algorithm. The steps are as follows.

Step 1: Parameters initialization. Define the number of particles as $s h u$ and the maximum number of iterations as $N$, where $N=$ times $* f p \_p$->size (). Randomly generate $s h u$ initial particles $X_{0}$ and velocity $V_{0}$ which are from -3 to 3 . The initial temperature is $\mathrm{T}$. The temperature control parameter is $r \_t$ and annealing probability $p$.

Step 2: Assume the best position of the particle itself is pre_cost and the global best position of particle swarm is best.

Step 3: Decide whether the current temperature reaches the final temperature term_temp. If achieved, then jump to Step 6, else jump to step 4.

Step 4: Do the following for all particles:

$\ominus$ Calculate the fitness of each particle according to Formula (1). If the particle adapt better than pre_cost or the random number is less than annealing probability $P$ which can be calculated by Formula (4), Formula (5) and Formula (6), then the value is assigned to pre_cost and update the best position of the individual particle. 
Table 1. Characteristics of the used testcases.

\begin{tabular}{|c|c|c|c|c|}
\hline Testcases & Number of IP/cores & $\begin{array}{c}\text { Core } \\
\text { avg. } \mathbf{W} / \mathbf{H}\end{array}$ & $\begin{array}{c}\text { Core std. dev. } \\
\text { of W/H }\end{array}$ & $\begin{array}{c}\text { Direct Topology } \\
\mathbf{R} \times \mathbf{R}\end{array}$ \\
\hline \hline apte & 8 & $4324 / 2499$ & $27 / 4$ & $3 \times 3$ \\
\hline xerox & 10 & $2114 / 2872$ & $335 / 1290$ & $4 \times 4$ \\
\hline hp & 11 & $4533 / 924$ & $2498 / 386$ & $1201 / 896$ \\
\hline ami25 & 25 & $1770 / 1408$ & $830 / 865$ & $6 \times 5$ \\
\hline ami33 & 33 & $1581 / 1573$ & $768 / 651$ & $6 \times 6$ \\
\hline ami49 & 49 & $1089 / 1123$ & & $7 \times 7$ \\
\hline
\end{tabular}

$\ominus$ If the fitness of pre_cost is better than best, then update the value of best and the value of goodnum adds to 1, else the value of badmum adds to 1 .

$\circledast$ Update the position and velocity of particles according to Formula (2) and Formula (3).

(4) Correct the obtained position information of particles according to the determined range of value of position and velocity so that it would not exceed the available space.

Step 5: Update temperature value according to Formulate (7) and jump to Step 3.

Step 6: Output best and end the algorithm.

\section{SIMULATION RESULTS AND DISCUSSION}

\subsection{Introduction of the Simulator}

In order to test the degree of improvement of the proposed algorithm on CPU processing time, average flit latency and network throughput, the algorithm needs to be run on an actual system and compared with the original algorithm.

In this paper, we use the VNOC3 simulation platform developed by Cristinel Ababei in North Dakota State University using $\mathrm{C}++$ in the Linux system. The simulator is actually a platform used to study $3 \mathrm{D}$ NoC architecture with two or three layers. The platform is built on a previous version of VNOC (a simulator for $2 \mathrm{D} \mathrm{NoC}$ ) and a $\mathrm{B}^{*}$ tree layout. For three-layer architecture, the framework uses hMetis division. In addition, the simulator also includes a hidden option which allows users to generate new testcases and a GUI drawing tools. The drawing tools can be used to generate a three-layer network architecture but with only 2D display.

\subsection{Testcases}

In our experiments, we used six testcases whose characteristics are shown in Table 1. In this table, we also present the size of the direct topologies for all the $3 \mathrm{~d}$ NoC testcases with heterogeneous network architecture. We constructed these testcases from the classic MCNC testcases, whose area was scaled to achieve an average size of about $1 \mathrm{~cm} \times 1 \mathrm{~cm}$, which is a typical area for NoCs reported in the literature [27-29].
Based on these six testcases we have done a lot of experiments and calculated the average as the experimental results. But due to the limitation of paper space, we show only three testcases of the six to illustrate trends and the improvement of the performance. They are apte, hp and ami49.

We choose apte and ami49 here since they respectively are the smallest and largest testcases in these six testcases and more persuasive. The testcase $h p$ is special, because the number of its IP core is relatively small (only 11). But its aspect ratio is 2 to 3 times the other testcases. So in this paper, we show comparison of 3 testcases.

\subsection{Simulation Results and Analysis}

With the simulator introduced above we implement PSOSA-NoC and compare with the original floorplanning algorithm based on simulated annealing algorithm to optimize the floorplans (hereinafter referred to as SA-NoC). In the simulation, the performance of the algorithm is mainly measured by three parameters, i.e. CPU processor time, throughput and average flit latency. In the simulation experiments, in order to ensure a fair comparison, the value of command line parameters (for example, operating cycle, preheat cycle, input and output buffer size, etc) of the two algorithm is always the same. In certain load conditions or certain Mesh size, the lower the average latency, higher the throughput, less the CPU processing time, the better performance of the algorithm.

In order to comprehensively study the impact of each indicator of 3D NoC on latency and throughput, in the simulation experiment, we compare the throughout and average latency from four aspects while other parameter values unchanged. (1) Change the number of virtual channels (from 2 to 6) and analyze the impact of virtual channels on average flit latency, throughput and CPU processing time. (2) Change the buffer size (from $1 \mathrm{x}$ to $5 \mathrm{x}$ and the data indicates 1 to 5 times the default cache) and analyze the impact of buffer size on average flit latency and throughput. (3) Change the injection load (20\%-100\%) and analyze the changes of CPU processing time, average flit latency and throughput. (4) Change Mesh size of the architecture (number of IP cores and aspect ratio) and analyze their impact on CPU processing, average flit latency and throughput. Detailed comparison and analysis are seen in section A, B, C, D. In the experiment, a number of simulations have been 


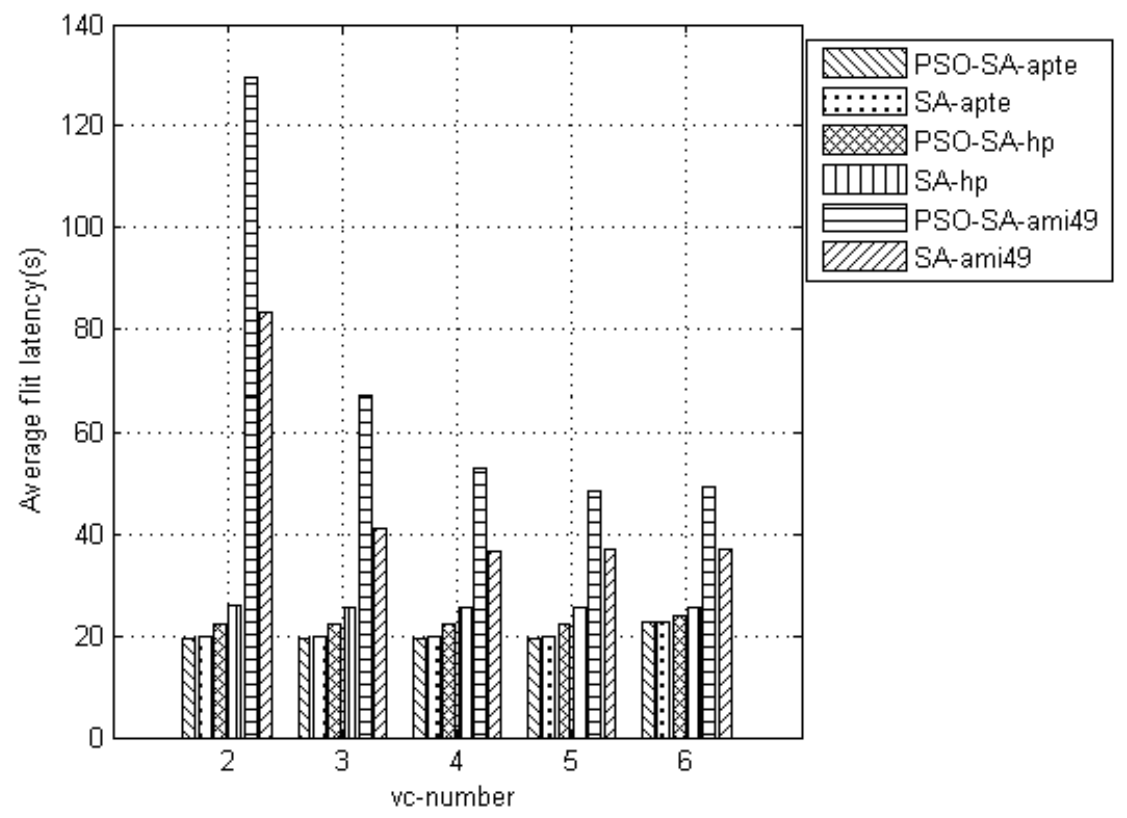

Fig. (1). Comparison of average flit latency for apte, $h p$ and ami49 while changing the number of virtual channels from 2 to 6.

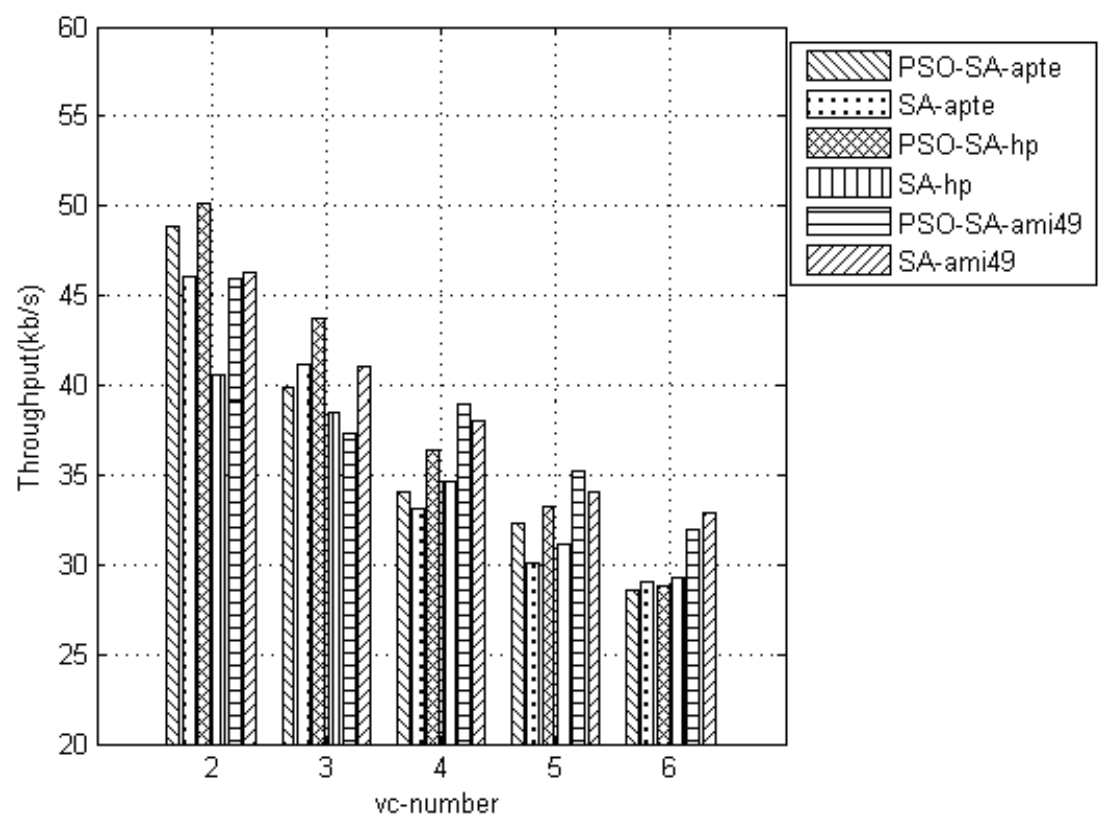

Fig. (2). Comparison of throughput for apte, $h p$ and ami49 while changing the number of virtual channels from 2 to 6.

conducted in each case and the final result is calculated from average. In each simulation, total run cycle is set to 60000 cycles and preheat cycle is set to 1000 cycles to ensure the obtained data values when the system reaches a steady state to reduce the error data obtained in the simulation.

\subsubsection{The Impact of the Number of Virtual Channels on Performance}

First, we observe the impact of increasing the number on virtual channels upon average flit latency, throughput and CPU processing time. In this scenario, we observe changes of average flit latency, throughput and CPU processing time while changing the number of virtual channels (VC) from 2 to 6 and compare the changes when using PSO-SA-NoC and SA-NoC.

For the testcases apte, $h p$ and ami49, the comparison of average flit latency, throughput and CPU processing time of PSO-SA-NoC and SA-NoC are shown in Figs. (1-3). From the three figures, we can observe that for the testcase apte, compared with SA-NoC algorithm, the average flit latency of PSO-SA-NoC algorithm decreases by $2.44 \%$ in average and the network throughput improves by $2.90 \%$ in average. The most obvious is that the CPU processing time decreases by $20.98 \%$ in average which saves energy greatly. 
For the testcase $h p$, with these three figures, we can conclude that compared with SA-NoC algorithm, the average flit latency of PSO-SA-NoC algorithm decreases by $13.41 \%$ in average and the network throughput improves by $9.57 \%$ in average. The most obvious is that the CPU processing time decreases by $75.81 \%$ in average which saves energy greatly.

For the testcase ami49, we can observe that compared with SA-NoC algorithm, the average flit latency of PSO-SANoC algorithm increases by $45.34 \%$ in average and the network throughput decreased by $1.31 \%$ in average. The CPU processing time decreases by $21.91 \%$ in average which saves energy greatly.

In summary, it can be seen that compared with the original SA-NoC, average flit latency and throughput of PSO-
SA-NoC are much better when the size of mesh is not very large and do not improved when the size of mesh is large. However, CPU processing time decreases greatly in all cases, by an average of $39.57 \%$ and the maximum case decreases $75.81 \%$.

\subsubsection{The Impact of Buffer Size on Performance}

In this scenario, instead of increasing the number of virtual channels, we study the impact of buffer size on performance by increasing the size of buffer. We assume that the areas of routers is about $20 \%$ of the total area, we can increase the areas of each router to reach to 5 times of the original value by increasing the size of input buffer and output buffer in each router port. We can observe the impact of

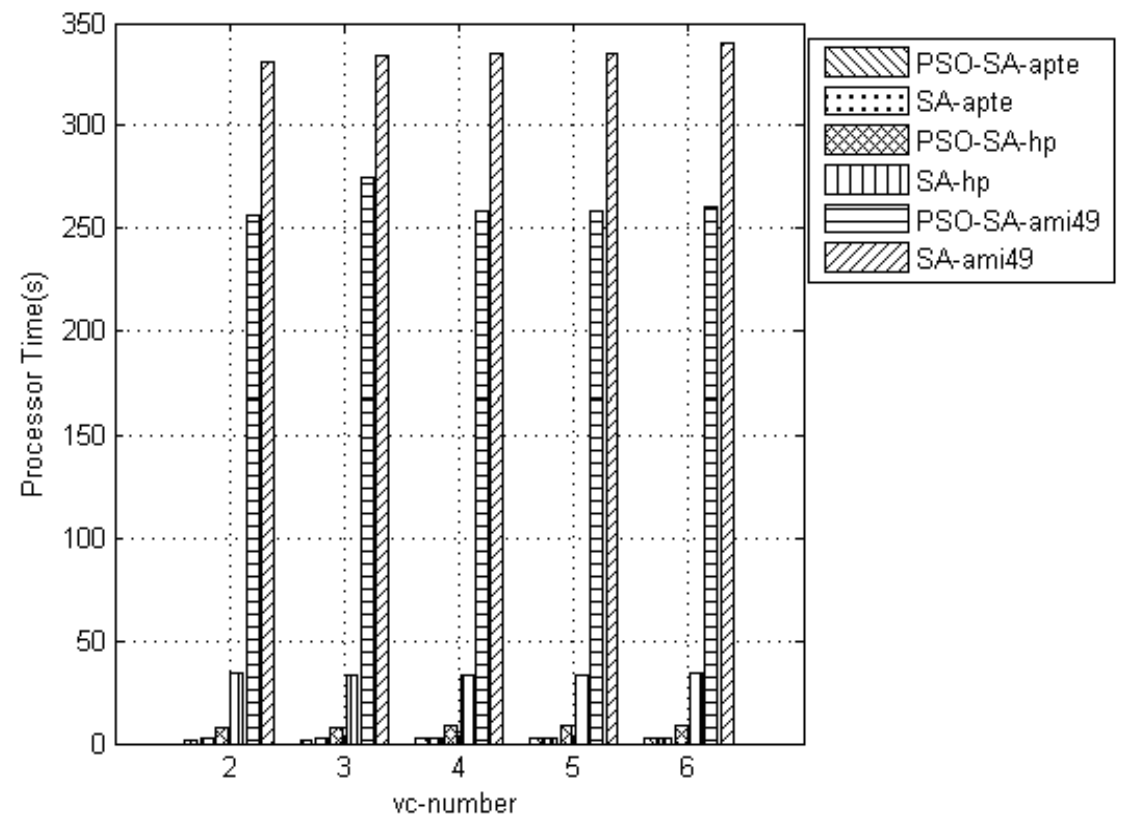

Fig. (3). Comparison of CPU processing time for apte, $h p$ and ami49 while changing the number of virtual channels from 2 to 6.

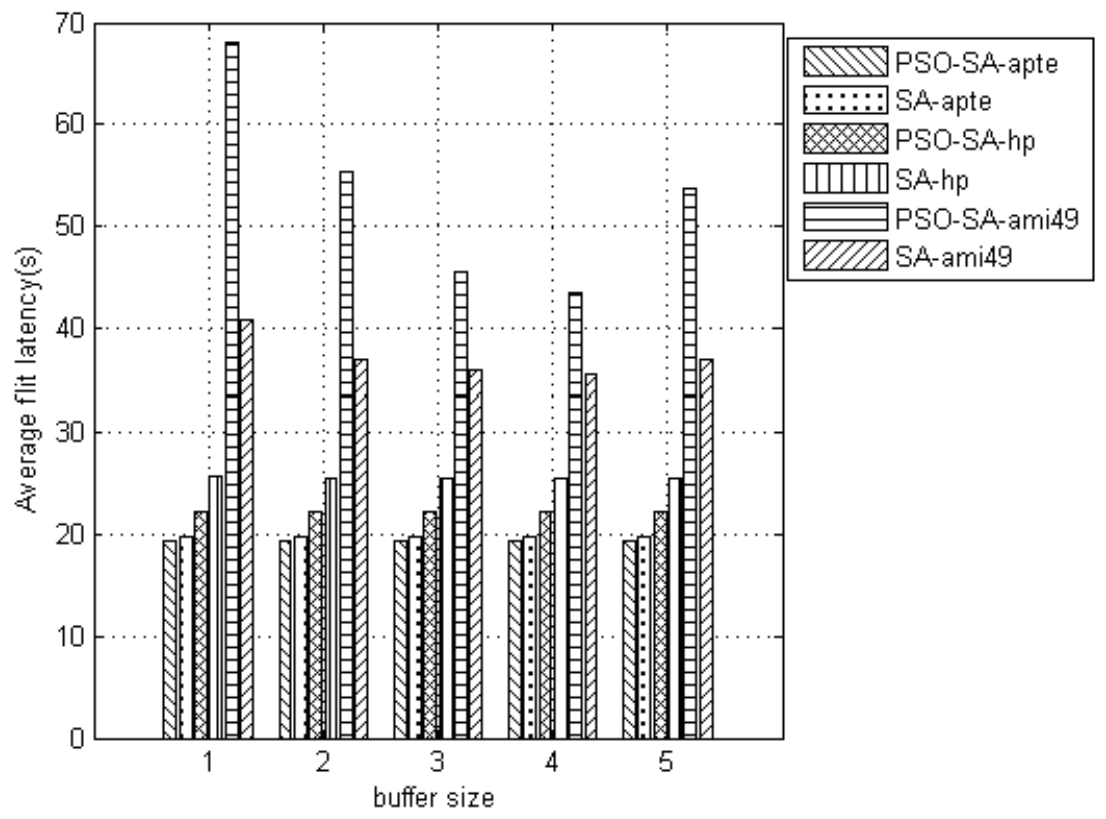

Fig. (4). Comparison of average flit latency for apte, $h p$ and ami49 while changing the size of buffers from $1 \mathrm{x}$ to $5 \mathrm{x}$. 
buffer size on average latency and throughput by changing the size of buffers.

For the testcases apte, $h p$ and ami49, the comparison of average flit latency and throughput of PSO-SA-NoC and SANoC are shown in Figs. (4 and 5). From the two figures, we can observe that for the testcase apte, compared with SA$\mathrm{NoC}$ algorithm, the average flit latency of PSO-SA-NoC algorithm decreases by $2.39 \%$ in average and network throughput improves by $4.36 \%$ in average.

For the testcase $h p$, with the two figures, we can conclude that compared with SA-NoC algorithm, the average flit latency of PSO-SA-NoC algorithm decreases by $13.07 \%$ in average and the network throughput improves by $50.64 \%$ in average. In this case, every performance of PSO-SA-NoC algorithm is much better than the original SA-NoC algorithm.

For the testcase ami49, we can observe that compared with SA-NoC algorithm, the average flit latency of PSO-SANoC algorithm increases by $41.98 \%$ in average and the network throughput decreased by $1.14 \%$ in average.

In summary, it can be seen that compared with the original SA-NoC, all performances of PSO-SA-NoC improve obviously in all cases when buffer size changes.

\subsubsection{The Impact of Injection Load on Performance}

Through a lot of experiments, we can conclude that the impact of changing the injection load on network latency and throughput is the largest. To compare the performance of the

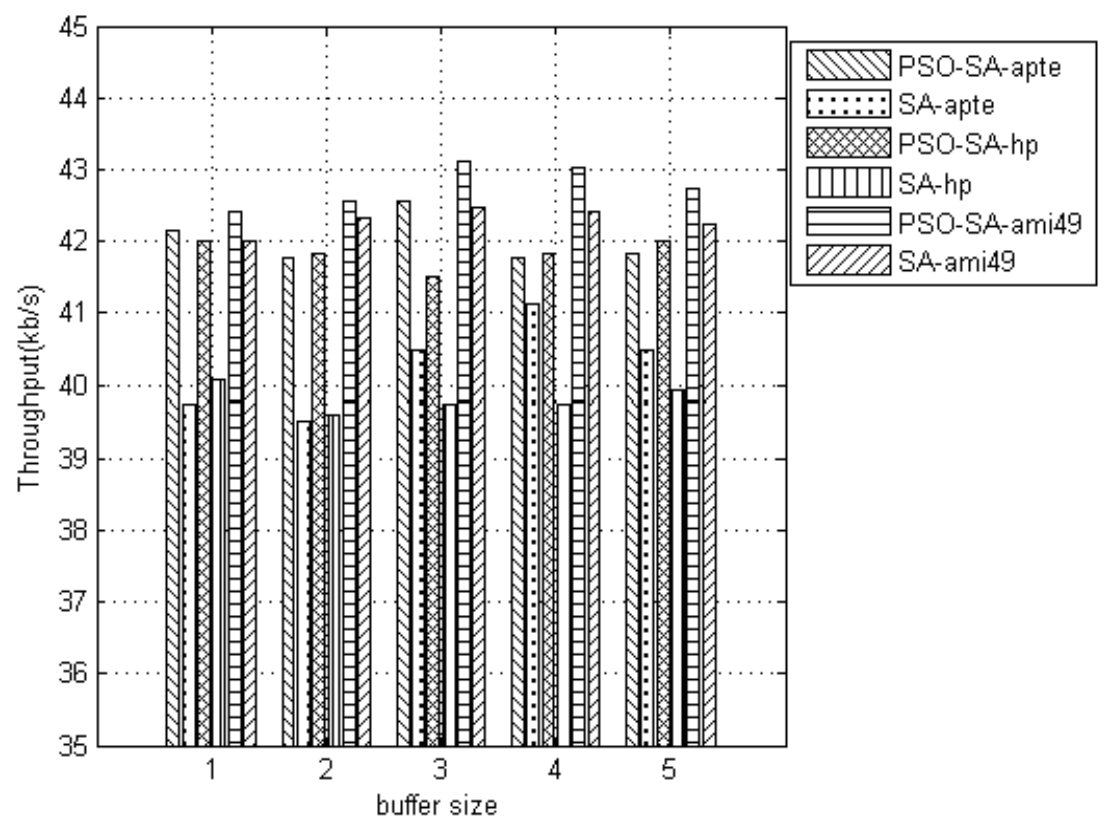

Fig. (5). Comparison of throughput for apte, $h p$ and ami49 while changing the size of buffers from $1 \mathrm{x}$ to $5 \mathrm{x}$.

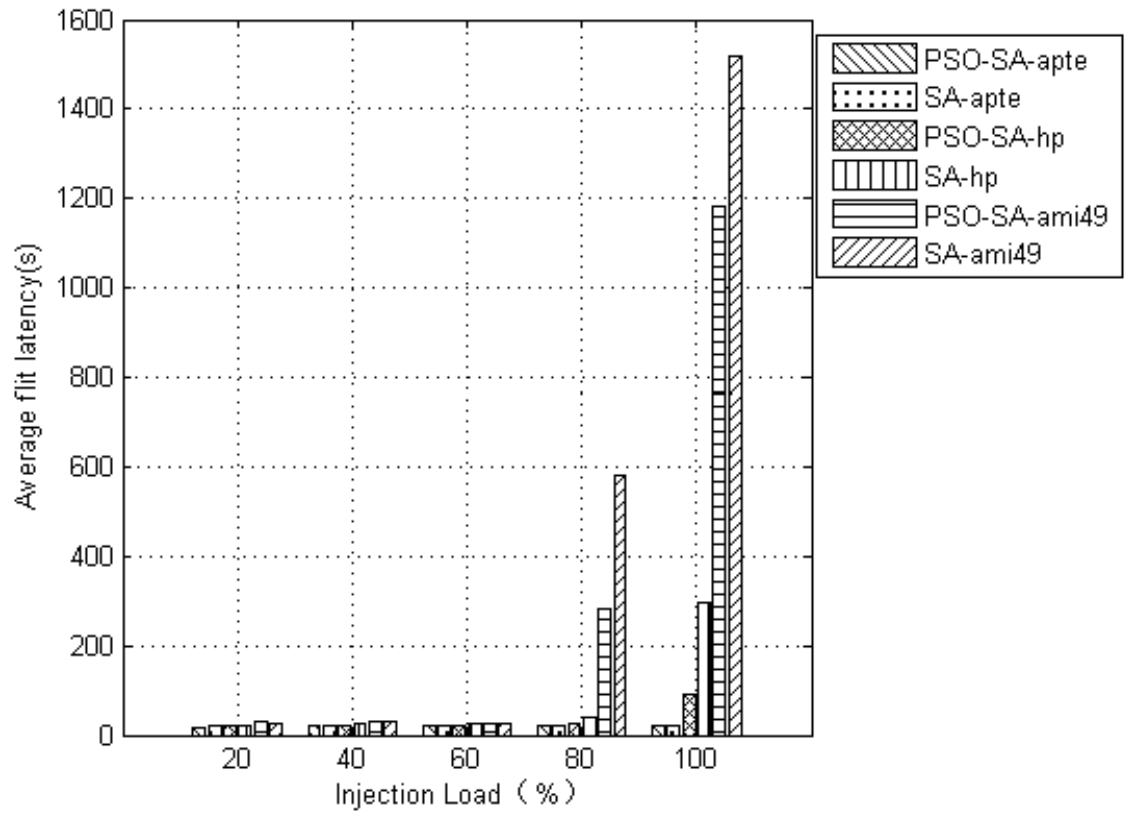

Fig. (6). Comparison of average flit latency for apte, $h p$ and ami49 while changing injection load. 


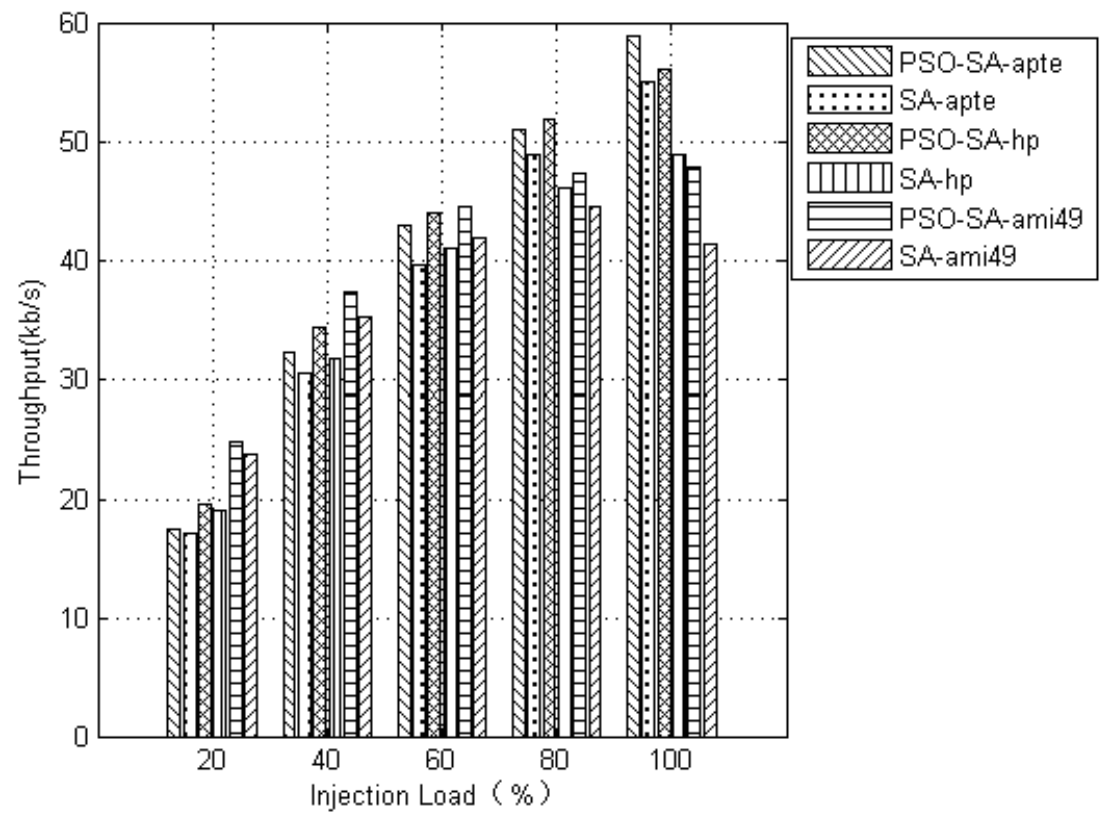

Fig. (7). Comparison of throughput for apte, $h p$ and ami49 while changing injection load.

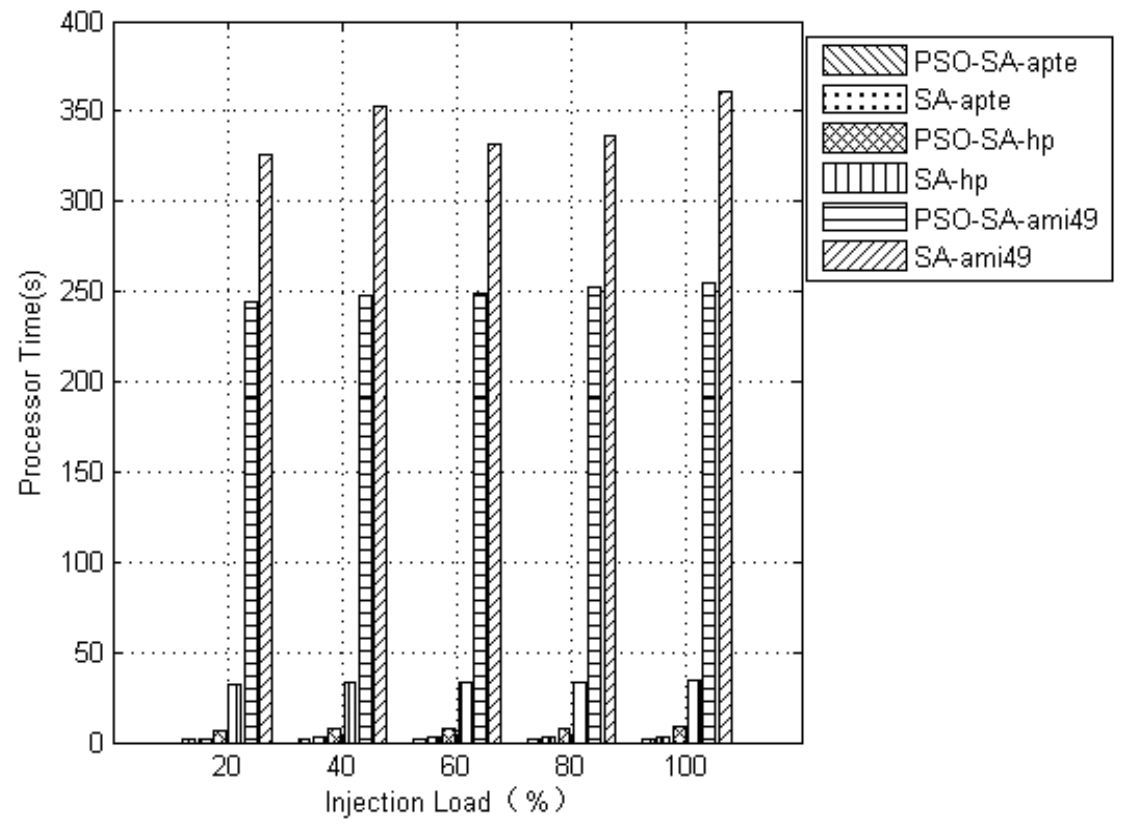

Fig. (8). Comparison of CPU processing time for apte, $h p$ and ami49 while changing injection load.

algorithm we proposed with the original algorithm, we need to explain that by changing injection load. In this paper, we conducted lots of experiments for the six testcases and eventually reach the following results by taking the average.

For the testcase apte, $h p$ and ami49, the comparison of average flit latency, throughout and CPU processing time of the two algorithms are shown in Figs. (6-8). From the three figures, we can observe that for the testcase apte, compared with SA-NoC algorithm, CPU processing time of PSO-SA$\mathrm{NoC}$ algorithm decreases by $25 \%$, the average flit latency decreases by $2.75 \%$ network throughput improves by $6.28 \%$.

For the testcase $h p$, before injection load reaches $70 \%$, average flit latency has no change substantially. With the three figures, we can conclude that compared with SA-NoC algorithm, CPU processing time of PSO-SA-NoC algorithm decreases by $77.24 \%$. Average flit latency increases by $28.33 \%$ and the network throughput improves by $9.77 \%$. In summary, for network architecture with smaller scale or larger aspect radio, PSO-SA-NoC algorithm improves performance better.

For the testcase ami49, we can observe that compared with SA-NoC algorithm, CPU processing time of PSO-SANoC algorithm decreases by $25.09 \%$. Average flit latency decreases by $18.89 \%$ and network throughput improves by $6.86 \%$. In this case, every performance of PSO-SA-NoC algorithm is better than the original SA-NoC algorithm. 


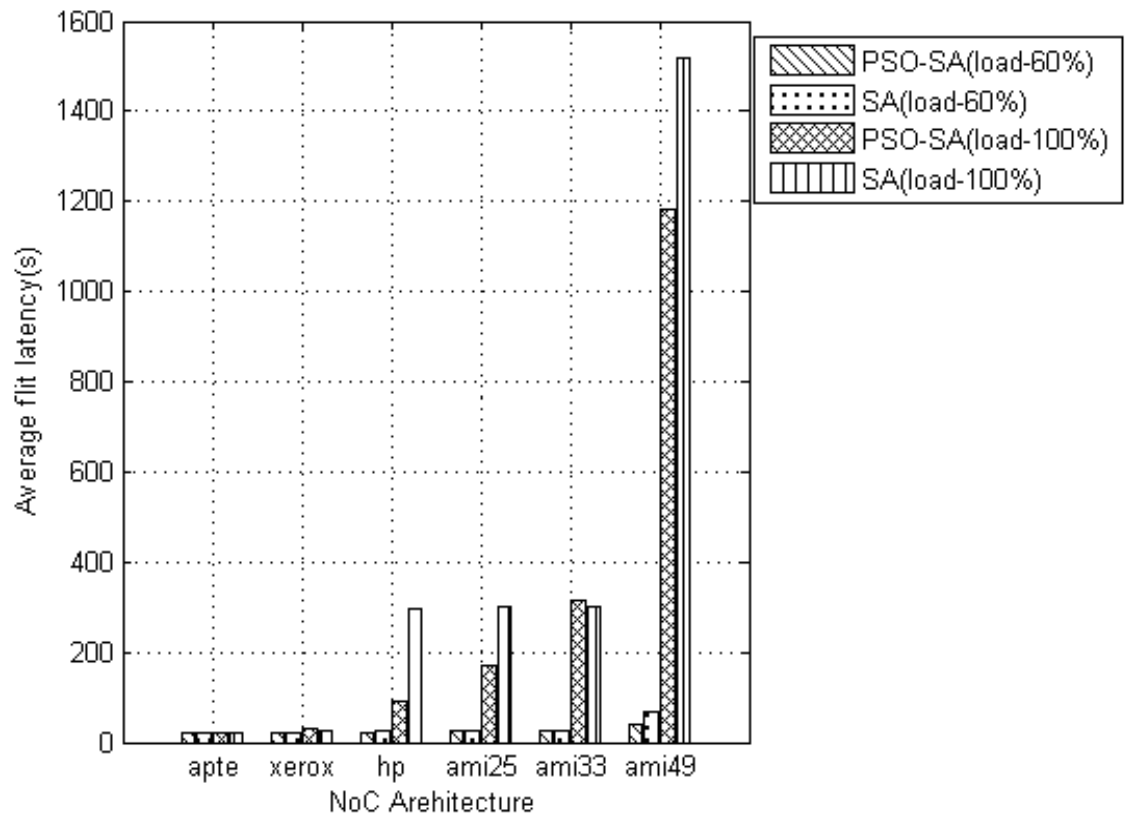

Fig. (9). Comparison of average flit latency of architectures with different Mesh size when injection load is $60 \%$ and $100 \%$.

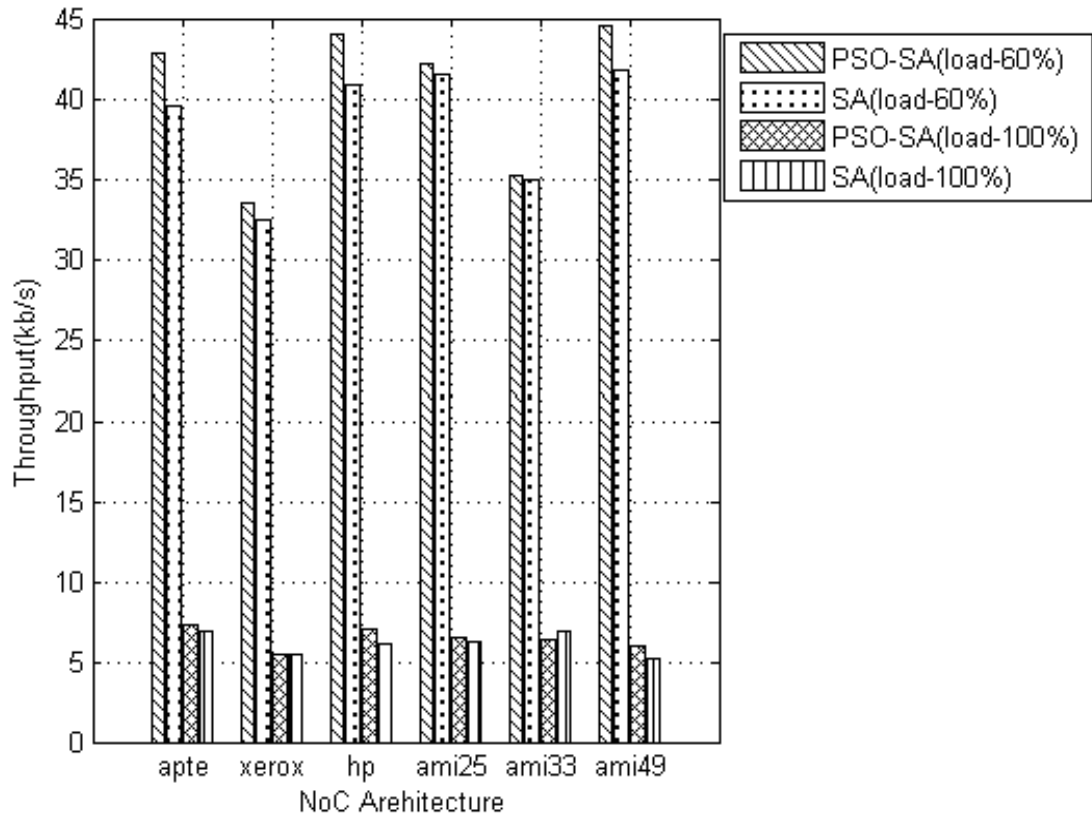

Fig. (10). Comparison of throughput of architectures with different Mesh size when injection load is $60 \%$ and $100 \%$.

In summary, it can be seen that compared with the original SA-NoC, CPU processing time of PSO-SA-NoC algorithm decreases greatly, by an average of $35.39 \%$ and the maximum case decreases $78.59 \%$. Average flit latency decreases $11.18 \%$ in average and $74.55 \%$ in the best case. Network throughput increases $6.17 \%$ in average and $24.02 \%$ in the best case.

\subsubsection{The Impact of Different Testcases on Performance}

Different testcases have different numbers of IP cores and different aspect ratio of the IP cores. Also, the Mesh size of their direct topologies is different. In this section, we study the change of average flit latency, throughput and CPU processing time of different testcases. In order to get more equitable results, in this paper, we do research and simulation of different injection loads and the results are as follows. When injection loads are different, the average flit latency increases as the number of IP cores increases, and the throughput is controlled by two parameters of Mesh size and aspect ratio. The variation trend of CPU processing time is substantially the same.

In this paper, we do simulation experiments of injection load of $20 \%$ to $100 \%$, and then we get the line chart and analyze it. But due to the limitations of our paper space, we just show the comparison of average flit latency in the condition of $60 \%$ and saturation injection load as shown in Fig. (9). It can be observed in the figure that when the Mesh size is less 


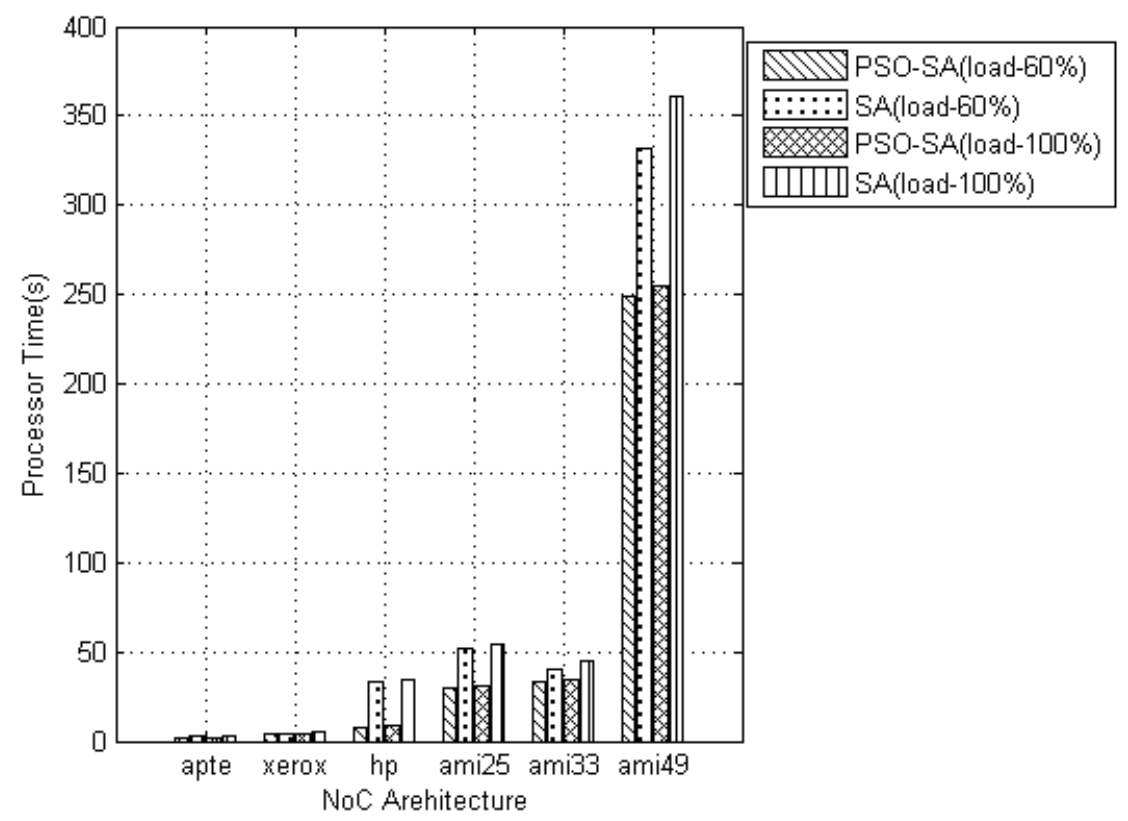

Fig. (11). Comparison of CPU processing time of architectures with different Mesh size when injection load is $60 \%$ and $100 \%$.

than 6, average flit latency increases more slowly and the average flit latency of PSO-SA-NoC algorithm is better than that of SA-NoC algorithm. When Mesh size is over 6, average flit latency of SA-NoC algorithm increases greater while that of the proposed PSO-SA-NoC algorithm increases slowly.

To illustrate the impact of different testcases on network throughput, in this paper, we also show the comparison of the impact of different Mesh size and respect ratio on throughput in the condition of $60 \%$ and saturation injection load as shown in Fig. (10). It can be observed in the figure that as a whole the throughput does not just increase or decrease as the number of IP cores increase, but shows a growth trend like a wavy line. This also explains why the throughput is not only affected by the number of IP cores but also the aspect ratio. According to the configuration parameters of each testcase shown in Table 1, we can draw a conclusion that the throughput is proportional to the number of IP cores and the aspect ratio. Therefore, although the number of IP cores of xerox increases, the aspect ratio decreases close to 1 . So the throughout presents a certain downward trend. For comparison of the two algorithms, the throughput of PSO-SA-NoC algorithm is slightly higher than that of SA$\mathrm{NoC}$ algorithm as a whole. In effect overall, PSO-SA-NoC algorithm is better.

For CPU processing time, in this paper, we just show the simulation results when the injection load is $60 \%$ and saturation injection load as shown in Fig. (11). It can be observed in the figure that when the Mesh size is less than 6, CPU processing time increases more slowly. When Mesh size is over 6, CPU processing time of SA-NoC algorithm increases at a speed of 7 times while that of PSO-SA-NoC algorithm increasing at a speed of 3 to 5 times. As a whole, compared with the original algorithm, CPU processing time of PSOSA-NoC algorithm decreases especially for architectures with large scale.
In summary, compared with SA-NoC algorithm, CPU processing time of PSO-SA-NoC algorithm decreases by $35.39 \%$. The network throughput increases and for architectures with Mesh size less than 6, the average flit latency decreases.

\section{CONCLUSION}

In this paper, we propose an improved floorplanning algorithm named the algorithm based on particle swarm optimization algorithm nesting simulated annealing algorithm to optimize the floorplans (PSO-SA-NoC). The algorithm is based on the advantages of parallel processing units of particle swarm optimization algorithm, and optimizes layout of the tiles to make the floorplanning path and the CPU processing time shorter and more efficient. Also, we adapt and build the original 3D NoC simulator to simulate the proposed algorithm and compare it with the original one. We add calculation method of throughput to make the experimental data more comprehensive and persuasive.

In the future, we need to design $\mathrm{NoC}$ architecture based on heterogeneous network architecture to replace original floorplanning algorithm. Secondly, it is helpful for a comprehensive study of architectures to write graphical interface that can display in three dimensions. In addition, 3D NoC architecture based on heterogeneous layout is from tree graph mapping. To ensure the quality of the mapping, researchers need to design better mapping algorithm to improve floorplanning performance of $3 \mathrm{D}$ NoC.

\section{CONFLICT OF INTEREST}

The authors confirm that this article content has no conflict of interest.

\section{ACKNOWLEDGEMENTS}

This work is supported by the National Natural Science Foundation of China (NSFC) (61272006). 


\section{REFERENCES}

[1] M. Sarrafzadeh, "Transforming an arbitrary floorplan into a sliceable one", In: Proceedings of the 1993 IEEE/ACM International Conference on Computer-Aided Design, Santa Clara, CA, USA, 1993.

[2] Y. Jin-Tai, L. Kai-Ping, and H. Chun-Tsai, "Sliceable transformation of non-slicing floorplans based on vacant block insertion in LB-packing process", In: IEEE International 48th Midwest Symposium on Circuits and Systems, Covington, KY, USA, 2005.

[3] Y. Jin-Tai, C. Chih-Wei, Y. -F. Luo, C. Yi-Hsiang, "Packingdriven sliceable transformation for 3D floorplan designs", In: Joint IEEE North-East Workshop on Circuits and Systems and TAISA Conference, Toulouse, France, 2008.

[4] O. Oluwaseun, "Parallel implementation of non-slicing floorplan with MPI and OpenMP", MS thesis, Ryerson University, 2012.

[5] Y. M. Li, "An non-slicing area prejudged algorithm for floorplanning without simulated annealing", In: 2nd International Conference on Computer and Automation Engineering, Singapore, 2010.

[6] C. Yu-Ning, "Non-slicing floorplanning-based crosstalk reduction on gridless track assignment for a gridless routing system with fast pseudo-tile extraction", In: ACM International Symposium on Physical Design, New York, NY, USA, 2008, pp. 134-141.

[7] X. Ning, "Hybrid algorithm for non-slicing floorplans optimization", In: 9th International Conference on Solid-State and Integrated-Circuit Technology, Beijing, 2008, pp. 2313-2316.

[8] H. -J. Bai, S. -Q. Dong, and X. - L. Hong, "Buffer insertion based on single-pair shortest-path algorithm for interconnect-centric floorplanning", In: 8th International Conference on Solid-State and Integrated Circuit Technology Proceedings, Shanghai, 2006, pp. 1873-1875.

[9] X. Hong, L. Ma, Y. Cai, C. K. Cheng, and J. Gu, "Sequence cloth diagram of Angle module and boundary constraint layout planning algorithm based on angle module expressed in sequence", Science China, vol. 32, no. 3, pp. 409-418, 2002.

[10] W. Haiqi, and D. Sheqin, "Topology generation algorithm for application specific network on chip", Journal of Computer Aided Design \& Computer Graphics, vol. 23, no. 9, pp. 1576-1584, 2011.

[11] H. Liang-li , W. Fa-yuan , and W. Feng-jun, "A method based on flock of birds with human-computer technology for packing layout", Journal of China Academy of Engineering Physics, vol. 3, pp. 29-31, 2009

[12] E. F. Y. Young, and T. Ma, "TCG-based multi-bend bus driven floorplanning", In: Proceedings of the Asia and South Pacific Design Automation Conference, Seoul, Korea, 2008, pp. 192-197.

[13] E. F. Y. Young, and R. Wang, "3-D floorplanning using labeled tree and dual sequences", In: Proceedings of the International Symposium on Physical Design, Seoul, Korea, 2008, pp. 54-59.

[14] J. He, "The Research and Development of Placement Algorithm for Network on Chip", PhD thesis, Wuhan University of Technology, 2010.
[15] K. M. Reza, A. Federico, M. Srinivasan, P. Antonio, S. Ciprian, and B. Luca, "A floorplan-aware interactive tool flow for NoC design and synthesis", In: Proceedings of IEEE International SOC Conference, Belfast, Northern Ireland, UK, 2009, pp. 379-382.

[16] V. De Paulo, and C. Ababei, "A framework for 2.5D NoC exploration using homogeneous networks over heterogeneous floorplans", In: International Conference on ReConFigurable Computing and FPGAs, Cancun, Quintana Roo, Mexico, 2009, pp. 267-272.

[17] D. Wang, J. Wang, and H. Wang, 'Intelligent Optimization Algorithms'. Higher Education Press, 2007, pp. 136-137.

[18] Y. Feng, "Research and application of simulated annealing algorithm", PhD thesis, Kunming University of Science and Technology, 2005, pp. 1-2.

[19] L. Wang, and Y. Qi, 'Application of simulated annealing algorithm to optimize the design of t-shaped micro-reactor', Computer Application and Chemistry, vol. 10, no. 30, pp. 1193-1196, 2013.

[20] Z. Liu, C. Liu, X. Kuang, and D. Zhou, "Application of simulated annealing algorithm to assessment of dynamic positioning capability", Journal of Ship Mechines, vol. 4, no. 17, pp. 375-381, 2013.

[21] J. Kennedy, and R. Eberhart, "Particle swarm optimization", In: IEEE International Conference on Neural Networks Proceedings, Perth, WA, Australia, 1995, pp. 1942-1948.

[22] R. Eberhart, and J. Kennedy, "A new optimizer using particle swarm theory", In: Proceedings of the 6th International Symposium on Micro Machine and Human Science, Nagoya, 1995, pp. 39-43.

[23] G. Q. Bao, and K. Mao, "An improved PSO algorithm and its utilization in wind power generation system", Control Engineering of China, vol. 20, no. 2, pp. 262-271, 2013.

[24] G. Ding, and W. Cao, "Application of improved particle swarm optimization algorithm in optimal operation of reservoir", South to North Water Transfers and Water Science \& Technology, vol. 12, no. 1, pp. 127-130, 2014.

[25] J. Zheng, "Research oil vehicle routing problem based onimproved particle swarm optimization algorithm", $\mathrm{PhD}$ thesis, North China Electric Power University, 2013, pp. 1-2.

[26] L. Jiang, "Study of BP network learning method based on particle swarm optimization algorithm and simulated annealing algorithm", $\mathrm{PhD}$ thesis, Anhui University, 2013, pp.1-2

[27] K. Kim, S. Lee, J. - Y. Kim, and M. Kim, "A $125 \mathrm{GOPS} 583 \mathrm{~mW}$ network-on-chip based parallel processor with bio-inspired visual attention engine", In: IEEE International Solid-State Circuits Conference, San Francisco, CA, 2008.

[28] S. R. Vangal, J. Howard, G. Ruhl, and S. Dighe, "An 80-Tile Sub100-W TeraFLOPS processor in 65-nm CMOS”, IEEE Journal Of Solid-State Circuits, vol. 43, no. 1, pp. 29-40, 2008.

[29] C. Mineo, R. Jenkal, and S. Melamed, "Inter-Die signaling in three dimensional integrated circuits", In: IEEE 2008 Custom Intergrated Circuits Conference, San Jose, CA, 2008, pp. 655-658.

(C) Guozhi et al.; Licensee Bentham Open.

This is an open access article licensed under the terms of the (https://creativecommons.org/licenses/by/4.0/legalcode), which permits unrestricted, noncommercial use, distribution and reproduction in any medium, provided the work is properly cited. 